Proc. Indian Acad. Sci. (Math. Sci.) Vol. 114, No. 1, February 2003, pp. 65-78.

Printed in India

\title{
On the limit-classifications of even and odd-order formally symmetric differential expressions
}

\author{
K V ALICE, V KRISHNA KUMAR* and A PADMANABHAN ${ }^{\dagger}$ \\ Department of Mathematics, Newman College, Thodupuzha 685 585, India \\ * Department of Mathematics, University of Calicut 673 635, India \\ ${ }^{\dagger}$ Department of Mathematics, Govt. College, Mokeri 673 516, India \\ E-mail: vellatkrishna@yahoo.com
}

MS received 10 July 2003

Abstract. In this paper we consider the formally symmetric differential expression $M[\cdot]$ of any order (odd or even) $\geq 2$. We characterise the dimension of the quotient space $D\left(T_{\max }\right) / D\left(T_{\min }\right)$ associated with $M[\cdot]$ in terms of the behaviour of the determinants

$$
\operatorname{det}_{r, s \in \mathbf{N}_{n}}\left[\left[f_{r} g_{s}\right](\infty)\right]
$$

where $1 \leq n \leq$ (order of the expression +1 ); here $[f g](\infty)=\lim _{x \rightarrow \infty}[f g](x)$, where $[f g](x)$ is the sesquilinear form in $f$ and $g$ associated with $M$. These results generalise the wellknown theorem that $M$ is in the limit-point case at $\infty$ if and only if $[f g](\infty)=0$ for every $f, g \in$ the maximal domain $\Delta$ associated with $M$.

Keywords. Limit classification, minimal and maximal closed operators; symmetric operators, self-adjoint operators; quotient space $D\left(T_{\max }\right) / D\left(T_{\min }\right)$.

\section{Introduction}

Let $\mathbf{N}$ denote the set of natural numbers and $\mathbf{N}_{k}:=\{1,2, \ldots, k\}$ for $k \in \mathbf{N}$. We write $C^{(r)}(I)(r=0,1,2, \ldots, m)$ for the class of complex valued functions defined on the interval $I$ with $r$ continuous derivatives, and $A C_{\text {loc }}(I)$ for the functions which are absolutely continuous on all compact sub intervals of $I$.

We consider the formally symmetric differential expression $M$ of order $m$ ( $m=$ $2 k$ or $2 k-1, k=1,2, \ldots$ ) given by (i.e. $M=M^{+}$, the formal Lagrange adjoint of $M$ )

$$
M[y]=\sum_{r=0}^{h}(-1)^{r}\left(s_{r} y^{(r)}\right)^{(r)}+\frac{1}{2} \sum_{r=0}^{k-1} i^{2 r+1}\left\{\left(q_{r} y^{(r)}\right)^{(r+1)}+\left(q_{r} y^{(r+1)}\right)^{(r)}\right\},
$$

where the sets of coefficients $\left\{s_{r}\right\}$ and $\left\{q_{r}\right\}$ are real valued on $I$ with $I$ designating the semi infinite interval $[0, \infty)$. Further we assume that

$$
\begin{array}{lr}
s_{r} \in C^{(r)}(I) & (r=0,1,2, \ldots, h) \\
q_{r} \in C^{(r+1)}(I) & (r=0,1,2, \ldots, k) .
\end{array}
$$

If $M$ is of even-order, $m=2 k(k=1,2, \ldots)$, then

$$
h=k \text { in (1.1) and } \quad s_{k}(x)>0, x \in I .
$$


If $M$ is of odd-order, $m=2 k-1(k=1,2, \ldots)$, then

$$
h=k-1 \text { in (1.1) and } q_{k-1}(x)>0, x \in I .
$$

Indeed, any formally symmetric differential expression of order $m$ with sufficiently smooth coefficients can be expressed as in (1.1) with suitable choice of coefficient functions.

The conditions (1.2) and (1.3) or (1.4) show that $M$ is regular on $[0, \infty)$, but $M$ has a singular point at $\infty$ (see [12], §15.1).

The differential equation we are concerned with is given by

$$
M[y]=\lambda y \quad \text { on } I,
$$

where $\lambda$ is a complex parameter $\lambda=\mu+i v$.

The standard existence theorems for ordinary, linear, homogeneous differential equations apply to eq. (1.5) (see the books: [2], ch. 3, $\S 6$ and [12], $\S 16.2$ ).

The underlying Hilbert space for the analysis of the problem is the collection of all equivalence classes of complex valued Lebesgue measurable functions $f$ on $[0, \infty)$ such that $\int_{0}^{\infty}|f|^{2}<\infty$ which is denoted by $L^{2}(0, \infty)$.

The Green's formula for $M$ takes the form

$$
\int_{0}^{x}\{\bar{g} M[f]-f \bar{M}[g]\}=[f g](x)-[f g](0), \quad x \in(0, \infty)
$$

where $f^{(m-1)}, g^{(m-1)} \in A C_{\mathrm{loc}}(0, \infty)$. Here the integrated term $[f g](x)$ on the R.H.S. is a skew-hermition, non-singular form on $[0, \infty)$ (see [2], ch. $3, \S 6$ and [8], §5).

Also

$$
[g f](x)=-[\overline{f g}](x), \quad x \in(0, \infty) .
$$

To set up the differential operators associated with $M[\cdot]$ in $L^{2}(0, \infty)$, we introduce the linear manifold $\Delta$ defined by

$$
\Delta:=\left\{f: f \in L^{2}(0, \infty), f^{(m-1)} \in A C_{\mathrm{loc}}(0, \infty) \text { and } M[f] \in L^{2}(0, \infty)\right\} .
$$

From an application of Green's formula we have

$$
[f g](\infty):=\lim _{x \rightarrow \infty}[f g](x)
$$

exists and is finite for all $f$ and $g$ in $\Delta$.

Next we introduce two differential operators $T_{\max }$ and $T_{\min }$, associated with $M$, defined as follows:

1. The maximal operator $T_{\max }$ : The domain $D\left(T_{\max }\right)$ is $\Delta$ and $T_{\max } f=M[f](f \in$ $\left.D\left(T_{\max }\right)\right)$

2. The minimal operator $T_{\min }$ : The domain $D\left(T_{\min }\right)$ is

$$
D\left(T_{\min }\right)=\{f: f \in \Delta \text { and }[f g](0)=[f g](\infty)=0 \text { for all } g \in \Delta\}
$$

and

$$
T_{\min } f=M[f] \quad\left(f \in D\left(T_{\min }\right)\right) .
$$


The domains $D\left(T_{\min }\right)$ and $D\left(T_{\max }\right)$ are dense in $L^{2}(0, \infty)$. These operators have the following properties:

(a) $T_{\min }$ is a closed, symmetric operator in $L^{2}(0, \infty)$.

(b) $T_{\max }$ is a closed, but not symmetric operator in $L^{2}(0, \infty)$.

(c) $T_{\min }^{*}=T_{\max }$; where $T_{\min }^{*}$ denotes the adjoint operator of $T_{\min }$ (see [3], ch. XIII, §2.12.8 and [12]).

The deficiency indices $\left(N_{+}, N_{-}\right)$of the closed, symmetric operator $T_{\min }$ are defined as

$$
N_{ \pm}=\operatorname{dim}\left\{f: f \in D\left(T_{\min }^{*}\right) ; T_{\min }^{*} f= \pm i f\right\} .
$$

From the general theory of deficiency indices of symmetric operators (see [3], ch. 12, $\S 19)$, we have

$$
\begin{gathered}
N_{ \pm}=\operatorname{dim}\left\{y \in C^{m}[0, \infty) ; M[y]=\lambda y \text { on }[0, \infty),\right. \\
\left.y \in L^{2}(0, \infty) \text { and } \lambda \in \mathbf{C}_{ \pm}\right\},
\end{gathered}
$$

where $C_{ \pm}=\{\lambda \in \mathbf{C}, \operatorname{Im} \lambda \gtrless 0\}$.

Thus the deficiency indices $N_{+}\left(N_{-}\right)$represent the number of linearly independent solutions of the differential equation (1.5) which are in $L^{2}(0, \infty)$ when $\lambda \in \mathbf{C}_{+}\left(\mathbf{C}_{-}\right)$. Hence both $N_{+}$and $N_{-}$are finite and

$$
0 \leq N_{ \pm} \leq m
$$

where $m$ is the order of the equation.

Further from the general theory of symmetric operators, it is known that $T_{\min }$ has selfadjoint extensions, i.e. $T_{\max }$ has self-adjoint restrictions in $L^{2}(0, \infty)$, if and only if $N_{+}=$ $N_{-}$. A better estimate of the lower bound for these indices in (1.7) are

1. When $m=2 k(k=1,2, \ldots)$ we get

$$
k \leq N_{+} \leq 2 k=m, k \leq N_{-} \leq 2 k=m .
$$

2. When $m=2 k-1(k=1,2, \ldots)$ we get

$$
k-1 \leq N_{+} \leq 2 k-1=m, k \leq N_{-} \leq 2 k-1=m
$$

(see [7] and [8]).

Any choice of integers $N_{+}\left(N_{-}\right)$satisfying (1.8) or (1.9) are possible pairs of deficiency indices provided they are also subject to the additional constraints $N_{+}=m$ if and only if $N_{-}=m$ for $m \geq 2$. For $m=1, N_{+}=0$ and $N_{-}=1$.

The differential expression $M$ is said to be in the limit $\left(N_{+}, N_{-}\right)$case at the singular point $\infty$ if the deficiency indices of the corresponding minimal closed operator $T_{\min }$ in $L^{2}(0, \infty)$ are $\left(N_{+}, N_{-}\right)$. In particular, borrowing the terminology of Weyl (see [2]) we say that $M$ is in the limit-point case at $\infty$, if $N_{+}=N_{-}=k$ in the even-order case and $N_{+}=k-1, N_{-}=k$ in the odd-order case.

We now introduce the Titchmarsh-Weyl $L^{2}(0, \infty)$ solutions of $(1.5)$ for the even- and odd-order cases. 
The even-order case

We assume $m=2 k(k \in \mathbf{N})$. Let $\theta_{r}$ and $\phi_{r}\left(r \in \mathbf{N}_{k}\right)$ be solutions of (1.5) taking initial values at 0 which are independent of $\lambda$, such that

$$
\left[\theta_{r} \theta_{s}\right](0)=0=\left[\phi_{r} \phi_{s}\right](0), \quad\left[\theta_{r} \phi_{s}\right](0)=\delta_{r s}, \quad r, s \in \mathbf{N}_{k},
$$

where $\delta_{r s}$ is the Kronecker delta function. Such a choice of initial conditions is possible and the set $\left\{\theta_{r}, \phi_{r} ; r \in \mathbf{N}_{k}\right\}$ forms a basis of solutions for (1.5). Then it can be seen that there are $k^{2}$ analytic functions $\left\{m_{r s}(\cdot) ; r, s \in \mathbf{N}_{k}\right\}$ which are all regular on $\mathbf{C}_{+} \cup \mathbf{C}_{-}$and such that the $k$ linearly independent solutions determined by

$$
\psi_{r}(x ; \lambda):=\theta_{r}(x ; \lambda)+\sum_{s=1}^{k} m_{r s}(\lambda) \phi_{s}(x ; \lambda), \quad\left(x \in[0, \infty), \lambda \in \mathbf{C}_{+} \cup \mathbf{C}_{-}\right)
$$

belong to $L^{2}(0, \infty)$ for $r \in \mathbf{N}_{k}$ and for all $\lambda \in \mathbf{C}_{+} \cup \mathbf{C}_{-}$. The analytic functions $m_{r s}(\lambda)$ satisfy

$$
m_{r s}(\lambda)=\bar{m}_{s r}(\bar{\lambda}), \quad\left(r, s \in \mathbf{N}_{k}\right)
$$

Thus it follows that to each $\lambda \in \mathbf{C}_{+} \cup \mathbf{C}_{-}$, there exist at least $k$ linearly independent solutions of (1.5) which belong to $L^{2}(0, \infty)$. The deficiency indices of the associated $T_{\min }$ can be characterised as

$$
N_{ \pm}=k+\operatorname{dim} \text { of the } L^{2}(0, \infty) \text { span of }\left\{\phi_{r}(\cdot, \pm i), r \in \mathbf{N}_{k}\right\} .
$$

For details, see [7].

The odd-order case

Here $m=2 k-1, k=2,3, \ldots$ Let $\theta_{r}(x ; \lambda)\left(x \in[0, \infty), \lambda \in \mathbf{C}, r \in \mathbf{N}_{k-1}\right)$ and $\phi_{s}(x, \lambda)(x \in$ $\left.[0, \infty), \lambda \in \mathbf{C}, s \in \mathbf{N}_{k}\right)$ be solutions of (1.5) taking initial values, independent of $\lambda$, at 0 such that

$$
\begin{aligned}
& {\left[\theta_{r} \theta_{s}\right](0)=0, \quad\left(r, s \in \mathbf{N}_{k-1}\right)} \\
& {\left[\theta_{r} \phi_{s}\right](0)=\delta_{r s}, \quad\left(r \in \mathbf{N}_{k-1}, s \in \mathbf{N}_{k}\right)} \\
& {\left[\boldsymbol{\phi}_{r} \phi_{s}\right](0)=i \delta_{r k} \delta_{s k}, \quad\left(r, s \in \mathbf{N}_{k}\right) .}
\end{aligned}
$$

Then the set of functions $\left\{\theta_{r}, \phi_{s} ; r \in \mathbf{N}_{k-1}, s \in \mathbf{N}_{k}\right\}$ forms a basis for all solutions of (1.5). Further there exists $k(2 k-1)$ analytic functions $\left\{p_{r s}(\cdot) ; r, s \in \mathbf{N}_{k}\right\}$ and $\left\{n_{r s}(\cdot) ; r \in \mathbf{N}_{k}, s \in\right.$ $\left.\mathbf{N}_{k-1}\right\}$ with $p_{r s}\left(n_{r s}\right)$ regular in $\mathbf{C}_{+}\left(\mathbf{C}_{-}\right)$such that if the solutions $\psi_{p, r}$ and $\psi_{n, r}$ are defined by

$$
\begin{aligned}
& \psi_{p, r}(x, \lambda)=\theta_{r}(x, \lambda)+\sum_{s=1}^{k} p_{r s}(\lambda) \phi_{s}(x, \lambda),\left(x \in[0, \infty), \lambda \in \mathbf{C}_{+}, r \in \mathbf{N}_{k-1}\right) \\
& \psi_{p, k}(x, \lambda)=\sum_{s=1}^{k} p_{k s}(\lambda) \phi_{s}(x, \lambda), \quad\left(x \in[0, \infty), \lambda \in \mathbf{C}_{+}\right)
\end{aligned}
$$


and

$$
\begin{aligned}
& \psi_{n, r}(x, \lambda)=\theta_{r}(x, \lambda)+\sum_{s=1}^{k-1} n_{r s}(\lambda) \phi_{s}(x, \lambda),\left(x \in[0, \infty), \lambda \in \mathbf{C}_{-}, r \in \mathbf{N}_{k-1}\right) \\
& \psi_{n, k}(x, \lambda)=\phi_{k}(x, \lambda)+\sum_{s=1}^{k-1} n_{k s}(\lambda) \phi_{s}(x, \lambda), \quad\left(x \in[0, \infty), \lambda \in \mathbf{C}_{-}\right)
\end{aligned}
$$

then $\psi_{p, r}(\cdot, \lambda) \in L^{2}(0, \infty)$ for $r \in \mathbf{N}_{k}, \lambda \in \mathbf{C}_{+}$and $\psi_{n, r}(\cdot, \lambda) \in L^{2}(0, \infty)$ for $r \in \mathbf{N}_{k}, \lambda \in \mathbf{C}_{-}$ with the possibility of $\psi_{p . k}(\cdot, \lambda)$ being a null solution of (1.5) in certain cases (see [10], $\S \S 2$ and 3$)$. The connection between the existence of the integrable square solutions and the deficiency indices of the associated $T_{\min }$ is that

$$
\begin{aligned}
& N_{+}=k-1+\operatorname{dim} L^{2}(0, \infty) \text { span of }\left\{\phi_{r}(\cdot, \lambda), r \in \mathbf{N}_{k}, \lambda \in \mathbf{C}_{+}\right\}, \\
& N_{-}=k+\operatorname{dim} L^{2}(0, \infty) \text { span of }\left\{\phi_{r}(\cdot, \lambda), r \in \mathbf{N}_{k-1}, \lambda \in \mathbf{C}_{-}\right\} .
\end{aligned}
$$

In both the even- and odd-order cases we have an elegant characterisation of the limitpoint cases in terms of the behaviour of the sesquilinear form $[f g](x) ; f, g \in \Delta$ as $x \rightarrow$ the singular point. We shall recall this result in the following theorem.

Theorem 1.1. Let $M$ be a formally symmetric differential expression of order $m$ ( $m=$ $2 k$ or $2 k-1, k=1,2, \ldots)$ given by $(1.1)$ on $[0, \infty)$ and the coefficients satisfy the conditions (1.2). A necessary and sufficient condition for $M$ to be in the limit-point case (i.e. limit $(k, k)$ in the $2 k$ th-order case, limit $(k-1, k)$ in the $(2 k-1)$ th-order case $)$ is that

$$
[f g](\infty)=\lim _{x \rightarrow \infty}[f g](x)=0 \text { for all } f \text { and } g \text { in } \Delta \text {. }
$$

(See [6] and [10].)

The object of this paper is to generalise this result. The generalisations are given by Theorems 2.1 and 2.2 for the even- and odd-order cases respectively. Before we state these generalisations in Theorems 2.1 and 2.2, we quote some known results which find repeated application in the proof of the main results.

Lemma 1.1.(A determinantal identity associated with $M)$. Let $\left\{f_{r}, g_{r}\right.$ for $r=1,2, \ldots, m+$ $1\}$ be any two sets of $(m+1)$ functions all in $\mathbf{C}^{(m)}[0, \infty)$. Then

$$
\operatorname{det}_{r, s \in \mathbf{N}_{m+1}}\left[\left[f_{r} g_{s}\right](x)\right]=0, \quad(x \in[0, \infty)),
$$

where $[f g](\cdot)$ is the sesquilinear form in $f$ and $g$ associated with $M$ (see [4], §11).

For the description of the system $\left\{f_{r}, g_{r}\right\}$ we use the following convention:

$$
\begin{aligned}
& f_{r}: f_{1}, f_{2}, \ldots, f_{m+1}, \\
& g_{r}: g_{1}, g_{2}, \ldots, g_{m+1},
\end{aligned}
$$

where $f_{r}, g_{r}$ correspond to the specific functions to be substituted in the identity.

Lemma 1.2.(the $L^{2}(0, \infty)$ lemma). Suppose that the complex valued measurable functions $f$ and $g$ on $[0, \infty)$ are such that $f \in L^{2}(0, \infty), g \in L^{2}(0, x),(x \in[0, \infty))$ and $g \notin L^{2}(0, \infty)$. Then

$$
\lim _{x \rightarrow \infty}\left\{\int_{0}^{x} f \bar{g}\right\}\left\{\int_{0}^{x}|g|^{2}\right\}^{-1 / 2}=0 .
$$

(See [6], §2.) 


\section{The main results}

The main results of this paper are as follows:

Theorem 2.1. Even-order case. Let $M$ be a formally symmetric differential expression of order $2 k(k=1,2, \ldots)$. Let $p$ and $q$ be non-negative integers such that $0 \leq p, q \leq k$. Let $n=p+q$. Then a necessary condition for $M$ to be in the limit $(k+p, k+q)$ case at $\infty$ is that

$$
\operatorname{det}_{r, s \in \mathbf{N}_{n+1}}\left[\left[f_{r} g_{s}\right](\infty)\right]=0 \quad \text { for all } f_{r}, g_{s} \in \Delta .
$$

Conversely, if

$$
\operatorname{det}_{r, s \in \mathbf{N}_{n+1}}\left[\left[f_{r} g_{s}\right](\infty)\right]=0 \quad \text { for all } f_{r}, g_{s} \in \Delta,
$$

then $M$ is in the limit $(k+p, k+q)$ case at $\infty$ where $p+q \leq n$. More precisely $p+q=n$ if (2.14) holds for all $f_{r}, g_{s} \in \Delta\left(r, s \in N_{n+1}\right)$ but

$$
\operatorname{det}_{r, s \in \mathbf{N}_{n}}\left[\left[f_{r} g_{s}\right](\infty)\right] \neq 0 \quad \text { for some } f_{r}, g_{s} \in \Delta .
$$

Remark 2.1. Here the choice of $p$ and $q$ are not unique. Any $p, q$ such that $0 \leq p, q \leq k$ is possible subject to the other constraints. For instance, in the case where $M$ is real, the deficiency indices are necessarily equal and hence $p=q$. Also whether $M$ is real or not, whenever $p=k$, then $q=k$. Such constraints do not come out from the theorem.

Theorem 2.2. Odd-order case. Let $M$ be a formally symmetric differential expression of order $2 k-1(k=2,3, \ldots)$. Let $p, q$ be non-negative integers such that $0 \leq p \leq k, 0 \leq q \leq$ $k-1$. Let $n=p+q$. Then a necessary condition for $M$ to be in the limit $(k-1+p, k+q)$ case at $\infty$ is that

$$
\operatorname{det}_{r, s \in \mathbf{N}_{n+1}}\left[\left[f_{r} g_{s}\right](\infty)\right]=0 \quad \text { for all } f_{r}, g_{s} \in \Delta .
$$

Conversely, if

$$
\operatorname{det}_{r, s \in \mathbf{N}_{n+1}}\left[\left[f_{r} g_{s}\right](\infty)\right]=0 \quad \text { for all } f_{r}, g_{s} \in \Delta,
$$

then $M$ is in the limit $(k-1+p, k+q)$ case at $\infty$ for some $p$ and $q$ such that $p+q \leq n$. More precisely $p+q=n$ if (2.16) holds for all $f_{r}, g_{s} \in \Delta\left(r, s \in \mathbf{N}_{n+1}\right)$ but

$$
\operatorname{det}_{r, s \in \mathbf{N}_{n}}\left[\left[f_{r} g_{s}\right](\infty)\right] \neq 0 \quad \text { for some } f_{r}, g_{s} \in \Delta .
$$

\section{Proof of the results}

Proof of Theorem 2.1. This is similar to the proof of Theorem 2.2, but less complicated. Therefore we omit the details (see [1], ch. 4).

We choose to give the proof of the theorem for the odd-order case in detail (see [13], ch. 5). 
Proof of Theorem 2.2.(Necessity). Suppose that $M$ is in the limit $(k-1+p, k+q)$ case at $\infty$; where $p+q=n$. Let $\lambda$ be a fixed point in $\mathbf{C}_{+}$and $\phi(\cdot)$ and $\tilde{\phi}(\cdot)$ denote $\phi(\cdot, \lambda)$ and $\phi(\cdot, \bar{\lambda})$ respectively. Then, since $M$ is assumed to be in the limit $(k-1+p, k+q)$ case at $\infty$, from (1.12) it is clear that there exist numbers $t_{1}, t_{2}, \ldots, t_{p} \in \mathbf{N}_{k}, t_{i} \neq t_{j}\left(i, j \in \mathbf{N}_{p}\right)$ such that the $L^{2}(0, \infty)$ spans of the $(k-p)$ functions $\left\{\phi_{s}(\cdot), s \neq t_{i}, i \in \mathbf{N}_{p}\right\}$ are null. Also there exist numbers $r_{1}, r_{2}, \ldots, r_{q}$ belonging to $\mathbf{N}_{k-1}, r_{i} \neq r_{j}\left(i, j \in \mathbf{N}_{q}\right)$ such that the $L^{2}(0, \infty)$ spans of the $(k-1-q)$ functions $\left\{\tilde{\phi}(\cdot) ; s \in \mathbf{N}_{k-1}, s \neq r_{i}, i \in \mathbf{N}_{q}\right\}$ are null.

Now we construct functions $\left\{\pi_{s}(x ; \cdot) ; s \in \mathbf{N}_{k-p}\right\}$ defined on $[0, x], x \in[0, \infty)$ from the set of functions $\left\{\phi_{s}(\cdot) ; s \in \mathbf{N}_{k}, s \neq t_{i}, i \in \mathbf{N}_{p}\right\}$ such that

$$
\pi_{s}(x ; \cdot)=\sum_{t=1}^{k-p} \alpha_{s t}(x) \phi_{t}(\cdot), \quad\left(s \in \mathbf{N}_{k-p}, x \in[0, \infty)\right)
$$

are $(k-p)$ linearly independent functions on $[0, x]$ and

$$
\int_{0}^{\pi} \pi_{s}(x ; \cdot) \bar{\pi}_{t}(x ; \cdot)=\mathbf{P}_{s}(x) \delta_{s t}, \quad\left(s, t \in \mathbf{N}_{k-p}, x \in[0, \infty)\right)
$$

where $0<\mathbf{P}_{1}(x) \leq \mathbf{P}_{2}(x) \leq \ldots \leq \mathbf{P}_{k-p}(x)<\infty$ and

$$
\lim _{x \rightarrow \infty} \mathbf{P}_{s}(x)=\infty . \quad\left(s \in \mathbf{N}_{k-p}\right)
$$

This is achieved by diagonalising the Gram matrix

$$
\Gamma_{x}=\left[\int_{0}^{x} \phi_{s}(\cdot) \bar{\phi}_{t}(\cdot)\right], \quad\left(s, t \in \mathbf{N}_{k-p}, x \in[0, \infty)\right)
$$

to $\operatorname{diag}\left(\mathbf{P}_{1}(x), \mathbf{P}_{2}(x), \ldots, \mathbf{P}_{k-p}(x)\right)$ through a unitary matrix $U_{x}=\left[\alpha_{s t}(x)\right], \quad\left(s \in \mathbf{N}_{k-p}, t \in\right.$ $\left.\mathbf{N}_{k-p}, x \in[0, \infty)\right) . \alpha_{s t}(x),(x \in[0, \infty))$ is the coefficient of $\phi_{t}(\cdot)$ in the definition (3.17) of $\pi_{s}(x ; \cdot)$. Note that (3.19) is a consequence of the fact that there exists no non-trivial linear combinations of $\left\{\phi_{s} ; s \in \mathbf{N}_{k-p}\right\} \in L^{2}(0, \infty)$. Proceeding along the same lines we construct functions $\left\{\sigma_{s}(x ; \cdot) ; s \in \mathbf{N}_{k-1-q}\right\}$ defined on $[0, x], x \in[0, \infty)$ from the set of functions $\left\{\tilde{\phi}_{s}(\cdot), s \in \mathbf{N}_{k-1}, s \neq r_{i}, i \in \mathbf{N}_{q}\right\}$ such that

$$
\sigma_{s}(x ; \cdot)=\sum_{t=1}^{k-1-q} \beta_{s t}(x) \tilde{\phi}_{t}(\cdot), \quad\left(s \in \mathbf{N}_{k-1-q}, x \in[0, \infty)\right)
$$

and

$$
\int_{0}^{x} \sigma_{s}(x ; \cdot) \bar{\sigma}_{t}(x ; \cdot)=Q_{s}(x) \delta_{s t}, \quad\left(s, t \in \mathbf{N}_{k-1-q}\right),
$$

where

$$
0<Q_{1}(x) \leq Q_{2}(x) \leq \ldots \leq Q_{k-1-q}(x)<\infty
$$

and

$$
\lim _{x \rightarrow \infty} Q_{s}(x)=\infty\left(s \in \mathbf{N}_{k-1-q}\right) .
$$


Then we apply Lemma 1.1 to the functions $\left\{f_{r}, g_{r}\right\}$ given by

$$
\begin{aligned}
& f_{r}: f_{1}, f_{2}, \ldots, f_{n+1}, \pi_{1}(x ; \cdot), \ldots, \pi_{k-p}(x ; \cdot), \sigma_{1}(x ; \cdot), \ldots, \sigma_{k-1-q}(x ; \cdot), \\
& g_{r}: g_{1}, g_{2}, \ldots, g_{n+1}, \pi_{1}(x ; \cdot), \ldots, \pi_{k-p}(x ; \cdot), \sigma_{1}(x ; \cdot), \ldots, \sigma_{k-1-q}(x ; \cdot),
\end{aligned}
$$

where $f_{r}, g_{s} \in \Delta\left(r, s \in \mathbf{N}_{n+1}\right)$.

In the resultant determinantal identity evaluated at $x(x \in[0, \infty))$ we divide the $(n+$ $1+r)$ th row and column by $\left\{P_{r}(x)\right\}^{1 / 2}$ and $(n+1+k-p+s)$ th row and column by $\left\{Q_{s}(x)\right\}^{1 / 2}$ for $\left(r \in \mathbf{N}_{k-p}, s \in \mathbf{N}_{k-1-q}\right)$. Then we obtain the following determinant identity given by

\begin{tabular}{|c|c|c|}
{$\left[f_{r} g_{s}\right](x)_{r, s \in \mathbf{N}_{n+1}}$} & $\frac{\left[\pi_{r} g_{s}\right](x)}{P_{r}^{1 / 2}}$ & $\frac{\left[\sigma_{r} g_{s}\right](x)}{Q_{r}^{1 / 2}}$ \\
\hline$\frac{\left[f_{r} \pi_{s}\right](x)}{P_{s}^{1 / 2}}$ & $\frac{\left[\pi_{r} \pi_{s}\right](x)}{P_{r}^{1 / 2} P_{s}^{1 / 2}}$ & $\frac{\left[\sigma_{r} \pi_{s}\right](x)}{Q_{r}^{1 / 2} P_{s}^{1 / 2}}$ \\
\hline$\frac{\left[f_{r} \sigma_{s}\right](x)}{Q_{s}^{1 / 2}}$ & $\frac{\left[\pi_{r} \sigma_{s}\right](x)}{P_{r}^{1 / 2} Q_{s}^{1 / 2}}$ & $\frac{\left[\sigma_{r} \sigma_{s}\right](x)}{Q_{r}^{1 / 2} Q_{s}^{1 / 2}}$
\end{tabular}$=0$.

Now we proceed to the limit as $x \rightarrow \infty$. We consider the limiting values of each of the terms in the above determinant. Note that

$$
\begin{aligned}
{\left[\pi_{r} \pi_{r}\right](x)=} & {\left[\pi_{r} \pi_{r}\right](0)+\int_{0}^{x}\left(\bar{\pi}_{r} M\left[\pi_{r}\right]-\pi_{r} \bar{M}\left[\pi_{r}\right]\right) } \\
& (\text { using the Green's formula) } \\
= & {\left[\pi_{r} \pi_{r}\right](0)+(\lambda-\bar{\lambda}) \int_{0}^{x} \pi_{r} \bar{\pi}_{r} } \\
= & {\left[\pi_{r} \pi_{r}\right](0)+2 i v \mathbf{P}_{r}(v=\operatorname{Im} \lambda) . }
\end{aligned}
$$

Therefore,

$$
\frac{\left[\pi_{r} \pi_{r}\right](x)}{\mathbf{P}_{r}}=\frac{\left[\pi_{r} \pi_{r}\right](0)}{\mathbf{P}_{r}}+2 i v .
$$

Now using (3.19) we get

$$
\lim _{x \rightarrow \infty} \frac{\left[\pi_{r} \boldsymbol{\pi}_{s}\right](x)}{\mathbf{P}_{r}}=2 i v .
$$

Also

$$
\begin{aligned}
{\left[\pi_{r} \pi_{s}\right](x) } & =\left[\pi_{r} \pi_{s}\right](0)+(\lambda-\bar{\lambda}) \int_{0}^{x} \pi_{r} \bar{\pi}_{s} \\
& =\left[\pi_{r} \pi_{s}\right](0), \quad \text { by }(3.18) .
\end{aligned}
$$

Therefore

$$
\lim _{x \rightarrow \infty} \frac{\left[\pi_{r} \pi_{s}\right](x)}{P_{r}^{1 / 2} P_{S}^{1 / 2}}=\lim _{x \rightarrow \infty} \frac{\left[\pi_{r} \pi_{s}\right](0)}{P_{r}^{1 / 2} P_{s}^{1 / 2}}=0 .
$$


Similarly, we get $\frac{\left[\sigma_{r} \sigma_{s}\right](x)}{Q_{r}^{1 / 2} Q_{s}^{1 / 2}}$ as $x \rightarrow \infty$.

$$
\begin{aligned}
{\left[f_{r} \pi_{s}\right](x) } & =\left[f_{r} \pi_{s}\right](0)+\int_{0}^{x}\left(\bar{\pi}_{s} M\left[f_{r}\right]-f_{r} \bar{M}\left[\pi_{s}\right]\right) \\
& =\left[f_{r} \pi_{s}\right](0)+\int_{0}^{x}\left(\bar{\pi}_{s} M\left[f_{r}\right]-f_{r} \bar{\lambda} \bar{\pi}_{s}\right) \\
& =\left[f_{r} \pi_{s}\right](0)+\int_{0}^{x} \bar{\pi}_{s}\left(M\left[f_{r}\right]-f_{r} \bar{\lambda}\right), \\
\lim _{x \rightarrow \infty} \frac{\left[f_{r} \pi_{s}\right](x)}{P_{s}^{1 / 2}} & =\lim _{x \rightarrow \infty} \frac{\left[f_{r} \pi_{s}\right](0)}{P_{s}^{1 / 2}}+\lim _{x \rightarrow \infty} \frac{\int_{0}^{x} \bar{\pi}_{s}\left(M\left[f_{r}\right]-f_{r} \bar{\lambda}\right)}{P_{s}^{1 / 2}} \\
& =0+\lim _{x \rightarrow \infty} \frac{\int_{0}^{x} \bar{\pi}_{s}\left(M\left[f_{r}\right]-f_{r} \bar{\lambda}\right)}{\left(\int_{0}^{x}\left|\pi_{s}\right|^{2}\right)^{1 / 2}} \\
& =0 .
\end{aligned}
$$

(Since $\pi_{s} \in L^{2}(0, x)$, but $\pi_{s} \notin L^{2}(0, \infty)$, we get the second term also as 0 by Lemma 1.2.) Similarly we can show that the terms

$$
\frac{\left[f_{r} \sigma_{s}\right](x)}{Q_{s}^{1 / 2}}, \frac{\left[\pi_{r} g_{s}\right](x)}{P_{r}^{1 / 2}}, \frac{\left[\sigma_{r} g_{s}\right](x)}{Q_{r}^{1 / 2}}
$$

tend to 0 as $x \rightarrow \infty$. Note that $\left[\pi_{r} \sigma_{s}\right](x)=\left[\pi_{r} \sigma_{s}\right](0)=0$. This follows from the properties of the fundamental solutions, given by (1.10). Therefore

$$
\lim _{x \rightarrow \infty} \frac{\left[\pi_{r} \sigma_{s}\right](x)}{P_{r}^{1 / 2} Q_{s}^{1 / 2}}=0 .
$$

Hence taking the limit, the determinant identity becomes

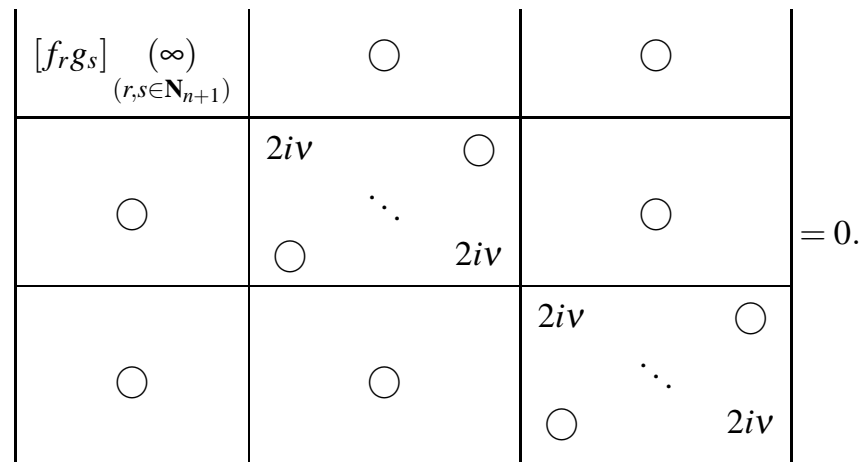

Since $v \neq 0, \operatorname{det}_{r, s \in \mathbf{N}_{n+1}}\left[\left[f_{r} g_{s}\right](\infty)\right]=0$ for all $f_{r}, g_{s} \in \Delta$. This completes the necessity part of the theorem. Now we prove the converse part of the theorem.

Remark 3.1. $\operatorname{det}_{r, s \in \mathbf{N}_{t}}\left[\left[f_{r} g_{s}\right](\infty)\right]=0$ for any $t<n+1 \Rightarrow \operatorname{det}_{r, s \in \mathbf{N}_{n+1}}\left[\left[f_{r} g_{s}\right](\infty)\right]=0$. Hence (2.16) can imply, if at all it is true, that $p+q \leq n$. Indeed, this is the case which we now prove in the converse part of the theorem. 
Sufficiency. Assume that (2.16) holds, then we show that $M$ cannot be in the limit $(k-$ $1+p, k+q)$ case at $\infty$ with $p+q>n$. To see this, we show that $M$ is in the limit $(k-1+$ $p, k+q$ ) case at $\infty$ with $p+q>n$ contradicting the validity of (2.16).

To be specific we assume that $p+q=n+1(p+q>n+1$ can be treated along the same lines).

For convenience we define

$$
\begin{array}{ll}
\psi_{r}:=\psi_{p, r}(x, \lambda), & \left(\lambda \in \mathbf{C}_{+}, r \in \mathbf{N}_{k-1}\right), \\
\tilde{\psi}_{r}:=\psi_{n, r}(x, \bar{\lambda}), \quad\left(\lambda \in \mathbf{C}_{+}, r \in \mathbf{N}_{k}\right),
\end{array}
$$

where $\psi_{p, r}, \psi_{n, r}$ are defined as in (1.11). Then for a given $\lambda \in \mathbf{C}_{+}$, in addition to the solutions $\psi_{1}, \psi_{2}, \ldots, \psi_{k-1}$, there exist $p$ solutions which are linear combinations of $\left\{\phi_{s}(\cdot, \lambda) ; s \in \mathbf{N}_{k}, \lambda \in \mathbf{C}_{+}\right\}$, say

$$
\begin{aligned}
& \xi_{t_{1}}(\cdot)=\phi_{t_{1}}(\cdot)+\sum_{\substack{s=1 \\
s \neq t_{1}}}^{k} \alpha_{1 s} \phi_{s}(\cdot), \quad\left(t_{1} \in \mathbf{N}_{k}\right), \\
& \xi_{t_{2}}(\cdot)=\phi_{t_{2}}(\cdot)+\sum_{\substack{s=1 \\
s \neq t_{1}, t_{2}}}^{k} \alpha_{2 s} \phi_{s}(\cdot), \quad\left(t_{2} \in \mathbf{N}_{k}\right)\left(t_{2} \neq t_{1}\right), \\
& \quad \vdots \\
& \xi_{t_{p}}(\cdot)=\phi_{t_{p}}(\cdot)+\sum_{\substack{s=1 \\
s \neq t_{1}, t_{2}, \ldots, t_{p}}}^{k} \alpha_{p s} \phi_{s}(\cdot), \quad\left(t_{p} \in \mathbf{N}_{k}\right)\left(t_{p} \neq t_{1}, t_{2}, \ldots, t_{p-1}\right)
\end{aligned}
$$

in $L^{2}(0, \infty)$ where $\left\{\alpha_{i s}, s \in \mathbf{N}_{k}, i \in \mathbf{N}_{p}, s \neq t_{i}\right\}$ are suitable complex numbers. Note that $\xi_{t}, i \in \mathbf{N}_{p}$ belong to $\Delta$.

Similarly for a given $\lambda \in \mathbf{C}_{+}$in addition to the solutions $\tilde{\psi}_{1}, \tilde{\psi}_{2}, \ldots, \tilde{\psi}_{k}$ belonging to $L^{2}(0, \infty)$, there exist $q$ solutions which are linear combinations of $\left\{\tilde{\phi}_{s}(\cdot), s \in \mathbf{N}_{k-1}, \lambda \in\right.$ $\left.\mathbf{C}_{+}\right\}$say

$$
\begin{aligned}
& \tilde{\eta}_{r_{1}}(\cdot)=\tilde{\phi}_{r_{1}}(\cdot)+\sum_{\substack{s=1 \\
s \neq r_{1}}}^{k-1} \beta_{1 s} \tilde{\phi}_{s}(\cdot), \quad\left(r_{1} \in \mathbf{N}_{k-1}\right), \\
& \tilde{\eta}_{r_{2}}(\cdot)=\tilde{\phi}_{r_{2}}(\cdot)+\sum_{\substack{s=1 \\
s \neq r_{1}, r_{2}}}^{k-1} \beta_{1 s} \tilde{\phi}_{s}(\cdot), \quad\left(r_{2} \in \mathbf{N}_{k-1}\right)\left(r_{2} \neq r_{1}\right), \\
& \vdots \\
& \tilde{\eta}_{r_{q}}(\cdot)=\tilde{\phi}_{r_{q}}(\cdot)+\sum_{\substack{s=1 \\
s \neq r_{1}, r_{2}, \ldots, r_{q}}}^{k-1} \beta_{1 s} \tilde{\phi}_{s}(\cdot), \quad\left(r_{q} \in \mathbf{N}_{k-1}\right)\left(r_{q} \neq r_{1}, r_{2}, \ldots, r_{q-1}\right)
\end{aligned}
$$

in $L^{2}(0, \infty)$ where $\left\{\beta_{j s}: j \in \mathbf{N}_{q}, s \in \mathbf{N}_{k-1}, s \neq r_{j}\right\}$ are suitable complex numbers. These solutions $\tilde{\eta}_{r_{j}}(\cdot), j \in \mathbf{N}_{q}$ belong to $\Delta$. 
Now consider the $(n+1) \times(n+1)$ determinant, $\operatorname{det}\left[\left[f_{r} g_{s}\right](\infty)\right]$ formed by choosing

$$
\begin{aligned}
& f_{r}: \xi_{t_{1}}(\cdot), \ldots, \xi_{t_{p}}(\cdot), \psi_{r_{1}}(\cdot), \psi_{r_{2}}(\cdot), \ldots, \psi_{r_{q}}(\cdot), \\
& g_{s}: \tilde{\psi}_{t_{1}}(\cdot), \ldots, \tilde{\psi}_{t_{p}}(\cdot), \tilde{\eta}_{r_{1}}(\cdot), \tilde{\eta}_{r_{2}}(\cdot), \ldots, \tilde{\eta}_{r_{q}}(\cdot) .
\end{aligned}
$$

This is given by

$$
\begin{array}{|c|c|}
{\left[\xi_{t_{i}} \tilde{\psi}_{t_{j}}\right]_{i, j \in \mathbf{N}_{p}}(\infty)} & {\left[\psi_{r_{i}} \tilde{\psi}_{t_{j}}\right]_{i \in \mathbf{N}_{q}, j \in \mathbf{N}_{p}}(\infty)} \\
\hline\left[\xi_{t_{i}} \tilde{\eta}_{r_{j}}\right]_{i \in \mathbf{N}_{p}, j \in \mathbf{N}_{q}}(\infty) & {\left[\psi_{r_{i}} \tilde{\psi}_{r_{j}}\right]_{i \in \mathbf{N}_{p}, j \in \mathbf{N}_{q}}(\infty)}
\end{array} .
$$

We evaluate this determinant (3.23) and show that it is not equal to zero. From Green's formula (1.6), it follows that all terms in the above determinant are finite. Further the value of each term of the determinant is its value at zero. This is because $[\xi \tilde{\eta}](x)$ is independent of $x$. Now we evaluate the determinant as follows. We consider the two cases separately.

Case 1. $t_{i} \neq k$ for every $i$. From the forms of $\xi_{t_{i}}(\cdot), \tilde{\eta}_{r_{j}}(\cdot)\left(i \in \mathbf{N}_{p}, j \in \mathbf{N}_{q}\right)$ in (3.20) and (3.21) respectively and the properties of $\left\{\theta_{r}\right\}$ and $\left\{\phi_{s}\right\}$ we get

$$
\begin{aligned}
& {\left[\xi_{t_{i}}(x) \tilde{\psi}_{t_{i}}(x)\right](\infty)=\left[\xi_{t_{i}}(x) \tilde{\psi}_{t_{i}}(x)\right](0)=-1 \quad\left(\forall i \in \mathbf{N}_{p}\right),} \\
& {\left[\xi_{t_{i}}(x) \tilde{\psi}_{t_{j}}(x)\right](\infty)=\left[\xi_{t_{i}}(x) \tilde{\psi}_{t_{j}}(x)\right](0)=0 \quad(\forall i>j),} \\
& {\left[\psi_{r_{i}}(x) \tilde{\psi}_{t_{j}}(x)\right](\infty)=\left[\psi_{r_{i}}(x) \tilde{\psi}_{t_{j}}(x)\right](0)=0 \quad(\forall i, j),} \\
& {\left[\xi_{t_{i}}(x) \tilde{\eta}_{r_{j}}(x)\right](\infty)=\left[\xi_{t_{i}}(x) \tilde{\eta}_{r_{j}}(x)\right](0)=0 \quad(\forall i, j),} \\
& {\left[\psi_{r_{i}}(x) \tilde{\eta}_{r_{j}}(x)\right](\infty)=\left[\psi_{r_{i}}(x) \tilde{\eta}_{r_{j}}(x)\right](0)=0 \quad(\forall i<j),} \\
& {\left[\psi_{r_{j}}(x) \tilde{\eta}_{r_{j}}(x)\right](\infty)=\left[\psi_{r_{j}}(x) \tilde{\eta}_{r_{j}}(x)\right](0)=1 \quad(\forall j) .}
\end{aligned}
$$

Therefore in this case we obtain the determinant as

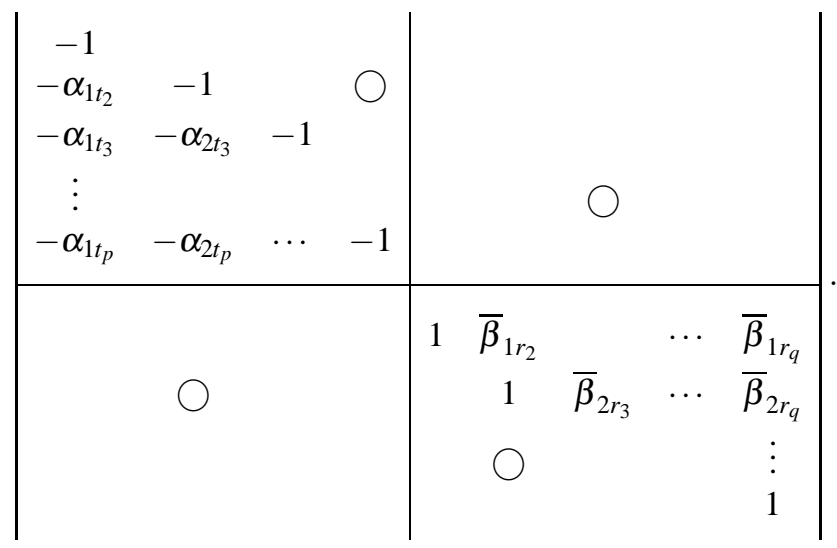

Case 2. $t_{i}=k$ for some $i$. We choose $t_{1}=k$ (there is no loss of generality in such a choice). 
Then we get,

$$
\begin{aligned}
& {\left[\xi_{t_{1}}(x) \tilde{\psi}_{t_{1}}(x)\right](\infty)=\left[\xi_{t_{1}}(x) \tilde{\psi}_{t_{1}}(x)\right](0)=i, \text { the imaginary unit, }} \\
& {\left[\xi_{t_{j}}(x) \tilde{\psi}_{t_{j}}(x)\right](\infty)=\left[\xi_{t_{j}}(x) \tilde{\psi}_{t_{j}}(x)\right](0)=-1 \quad(\forall j \geq 2),} \\
& {\left[\psi_{t_{i}}(x) \tilde{\psi}_{t_{j}}(x)\right](\infty)=\left[\psi_{t_{i}}(x) \tilde{\psi}_{t_{j}}(x)\right](0)=0 \quad(\forall i>j),} \\
& {\left[\psi_{r_{i}}(x) \tilde{\psi}_{t_{j}}(x)\right](\infty)=\left[\psi_{r_{i}}(x) \tilde{\psi}_{t_{j}}(x)\right](0)=0 \quad(\forall i, j),} \\
& {\left[\xi_{t_{i}}(x) \tilde{\eta}_{r_{j}}(x)\right](\infty)=\left[\xi_{t_{i}}(x) \tilde{\eta}_{r_{j}}(x)\right](0)=0 \quad(\forall i, j),} \\
& {\left[\psi_{r_{i}}(x) \tilde{\eta}_{r_{j}}(x)\right](\infty)=\left[\psi_{r_{i}}(x) \tilde{\eta}_{r_{j}}(x)\right](0)=0 \quad(\forall i<j),} \\
& {\left[\psi_{r_{j}}(x) \tilde{\eta}_{r_{j}}(x)\right](\infty)=\left[\psi_{r_{j}}(x) \tilde{\eta}_{r_{j}}(x)\right](0)=1 \quad(\forall j) .}
\end{aligned}
$$

Therefore in this case the determinant (3.23) takes the form

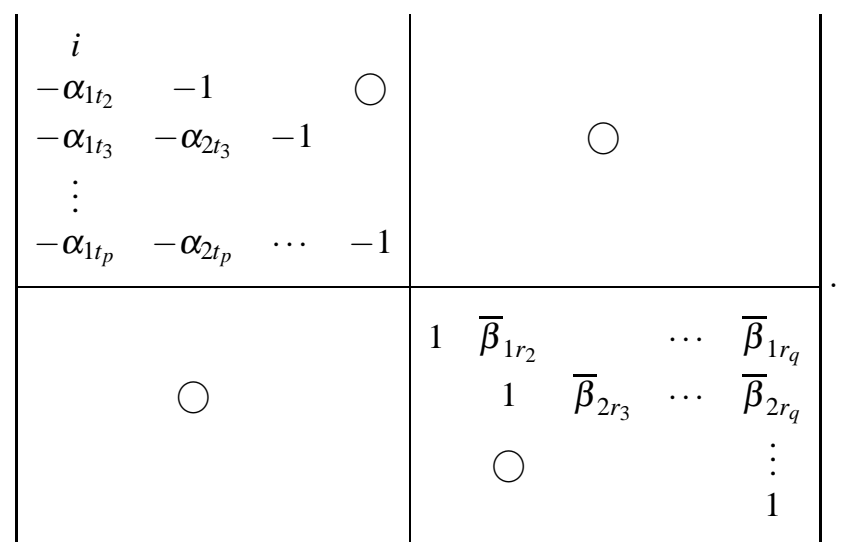

Thus in both the cases we get

$$
\underset{r, s \in \mathbf{N}_{n+1}}{\operatorname{det}}\left[\left[f_{r} g_{s}\right](\infty)\right] \neq 0,
$$

where $\left\{f_{r}\right\}$ and $\left\{g_{s}\right\}$ are as given in (3.22). This contradicts hypothesis (2.16). Hence if det $\left[\left[f_{r} g_{s}\right](\infty)\right]=0$ for all $f_{r}, g_{s} \in \Delta$, then $M$ is in the limit $(k-1+p, k+q)$ case at $\infty$ where $p+q \leq n$.

Remark 3.2. In the even-order case, the situation of Case 2 does not arise; this part of the proof follows as in Case 1 (see [1], ch. 4).

Next we prove that $p+q=n$ if (2.16) holds for all $f_{r}, g_{s} \in \Delta\left(r, s \in \mathbf{N}_{n+1}\right)$ but $\underset{r, s \in \mathbf{N}_{n}}{\operatorname{det}}\left[\left[f_{r} g_{s}\right](\infty)\right] \neq 0$ for some $f_{r}, g_{s} \in \Delta$. Assume that $\operatorname{det}_{r, s \in \mathbf{N}_{n+1}}\left[\left[f_{r} g_{s}\right](\infty)\right]=0$ for all $f_{r}, g_{s} \in \Delta$. This $\Rightarrow M$ is in the limit $(k-1+p, k+q)$ case at $\infty$, for some $p$ and $q$ such that $p+q \leq n$.

Further assume that $\operatorname{det}_{r, s \in \mathbf{N}_{n}}\left[\left[f_{r} g_{s}\right](\infty)\right] \neq 0$ for some $f_{r}, g_{s} \in \Delta$. From the necessity part this $\Rightarrow M$ is in the limit $(k-1+p, k+q)$ case at $\infty$ where $p+q \geq n$. Together, it now follows that $M$ is in the limit $(k-1+p, k+q)$ case at $\infty$ where $p+q=n$. 


\section{Concluding remarks}

1. When $p=0, q=0$, we recover the limit-point characterisation in Theorem (1.1). Therefore this result is a generalisation of the characterisation for the limit-point case at $\infty$.

2. Note that, $2 k-1+n(k-1+p+k+q)$ gives the gap between $D\left(T_{\min }\right)$ and $D\left(T_{\max }\right)$. More precisely if $\left(N_{+}, N_{-}\right)$gives the deficiency indices of $T_{\min }, N_{+}+N_{-}$gives the dimension of the quotient space $D\left(T_{\max }\right) / D\left(T_{\min }\right)$. Therefore the above result characterises the gap between $D\left(T_{\max }\right)$ and $D\left(T_{\min }\right)$. However, since $p+q$ and not $p$ and $q$ separately appear in the theorem the exact break up of the deficiency indices $(k-1+p, k+q)$ is not characterised by this result.

3. In the second-order case, this result has been made use of to obtain sufficient conditions on the coefficients for $M$ to be not in the limit-point case at $\infty$ [9].

4. In the third-order case also, this result has been used to obtain sufficient conditions on the coefficients for $M$ to be not in the limit-point case at $\infty$ [11].

In the third-order case, the admissible limit classifications of $M$ are as follows: $M$ is either in the limit $(1,2)$ case or in the $(2,2)$ case, or in the limit $(3,3)$ case at $\infty$. The above theorem takes the following forms in these cases:

i. $M$ is in the limit $(1,2)$ case at $\infty \Leftrightarrow[f g](\infty)=0, \quad \forall f, g \in \Delta$.

ii. $M$ is in the limit $(2,2)$ case at

$$
\infty \Leftrightarrow\left|\begin{array}{ll}
{\left[f_{1} g_{1}\right]} & {\left[f_{2} g_{2}\right]} \\
{\left[f_{2} g_{1}\right]} & {\left[f_{2} g_{2}\right]}
\end{array}\right|(\infty)=0
$$

and there exist $f, g \in \Delta$ such that $[f g](\infty) \neq 0$.

iii. $M$ is in the limit $(3,3)$ case at

$$
\infty \Leftrightarrow \operatorname{det}_{r, s \in \mathbf{N}_{4}}\left[\left[f_{r} g_{s}\right](\infty)\right]=0, \quad \forall f_{r}, g_{s} \in \Delta
$$

and there exists $f_{r}, g_{s} \in \Delta$ such that

$$
\operatorname{det}_{r, s \in \mathbf{N}_{3}}\left[\left[f_{r} g_{s}\right](\infty)\right] \neq 0 .
$$

Since (4.24) holds irrespective of the limit classifications of $M$ (Lemma 1.1), the required condition reduces to (4.25).

5. We hope that the criteria (i), (ii) and (iii) above may be made use of to obtain sufficient conditions on the coefficients of $M$ in the third-order case for $M$ to be in the limit $(3,3)$ case at $\infty$.

6. Scope for the application of this result to obtain conditions on the coefficients for specific limit classifications is very much limited in equations of order higher than 3 . For instance, consider the real, fourth-order case. Here the possible cases are limit $(2,2)$, limit $(3,3)$ and limit $(4,4)$. Now we can prove that $M$ is in the limit $(4,4)$ case at $\infty$, if we can construct functions $f_{1}, f_{2}, f_{3} \in \Delta$ such that

$$
\left|\begin{array}{lll}
{\left[f_{1} f_{1}\right]} & {\left[f_{2} f_{1}\right]} & {\left[f_{3} f_{1}\right]} \\
{\left[f_{1} f_{2}\right]} & {\left[f_{2} f_{2}\right]} & {\left[f_{3} f_{2}\right]} \\
{\left[f_{1} f_{3}\right]} & {\left[f_{2} f_{3}\right]} & {\left[f_{3} f_{3}\right]}
\end{array}\right|(\infty) \neq 0 .
$$


But this is not an easy task. Also to make use of it in the positive direction, it is even more difficult. This is so since we have to show that an $n \times n$ determinant vanishes at the singular point for every $f_{r}, g_{s} \in \Delta$ for large $n$.

7. If the BVP considered is $M y=\lambda w y$ with a positive weight function $w$ in $[0, \infty)$ we have to set the operators in $L_{w}^{2}(0, \infty)$, the weighted Hilbert space of integrable square function with weight $w$ on $[0, \infty)$. The related operators are to be defined from $\frac{1}{w} M[\cdot]$ acting over functions in $L_{w}^{2}(0, \infty)$. The sesquilinear form $[f g](x)$ remains the same, but the functions $f$ and $g$ are now to be chosen in $L_{w}^{2}(0, \infty)$. The analysis goes through with no significant change.

8. We have discussed the problem on the semi-infinite interval $[0, \infty)$. However, the analysis goes through without any significant changes, if it is considered on an interval $[a, b)$ with $-\infty<a<b \leq \infty$ where $b$ is a singular end-point, i.e., we shall assume that differential expression is regular on all compact sub-intervals of $[a, b)$ and is singular at $b$.

\section{Acknowledgements}

One of the authors (KVA) acknowledges the financial assistance received from the University of Calicut and the University Grants Commission, India during the work. AP acknowledges the financial assistance received from the University of Calicut and the Council of Scientific and Industrial Research, India during the work.

\section{References}

[1] Alice K V, On the symmetric differential operators, M.Phil. thesis (University of Calicut) (1987)

[2] Coddington E A and Levinson N, Theory of ordinary differential equations (New York and London: McGraw Hill) (1955)

[3] Dunford N and Schwartz J T, Linear operators, Part II (New York: Interscience) (1963)

[4] Everitt W N, Self-adjoint boundary value problems on finite intervals, J. London Math. Soc. 37 (1962) 372-384

[5] Everitt W N, Integrable-square solutions of ordinary differential equations (II), Quart. J. Math. Oxford (2) 13 (1962) 217-220

[6] Everitt W N, A note on the self-adjoint domains of second-order differential equations, Quart. J. Math. Oxford (2) 14 (1963) 41-45

[7] Everitt W N, Integrable-square solutions of ordinary differential equations (III), Quart. J. Math. Oxford (2) 14 (1963) 170-180

[8] Everitt W N, Integrable-square analytic solutions of the odd-order formally symmetric differential equations, Proc. London Math. Soc. (3) 25 (1972) 156-182

[9] Everitt W N, The limit-circle classification of second-order differential expressions, Quart. J. Math. Oxford (2) 23 (1972) 193-196

[10] Everitt W N and Krishna Kumar V, On the Titchmarsh-Weyl theory of ordinary symmetric differential equations I; General theory, Nieuw-Archief V. Wiskunde (3) XXIV (1976) 1-48

[11] Krishna Kumar V and Padmanabhan A, On the deficiency indices of third-order differential operators, Bull. London Math. Soc. 20 (1988) 333-336

[12] Naimark M A, Linear differential operators Part II (New York: Ungar) (1968), translated from Russian

[13] Padmanabhan A, On the deficiency index problem for odd-order symmetric differential operators, Ph.D. thesis (University of Calicut) (1988) 